\title{
Analysis of Efficient Storage Technique for Financial Data in Cloud
}

\author{
Rima Mihir Shah \\ Assistant Professor \\ Master of Computer Application \\ ITM Universe, \\ Vadodara, India \\ Dr. Priti Srinivas Sajja \\ Professor, Post Graduate Department of Computer Science \\ Sardar Patel University, \\ V.V.Nagar, India
}

\begin{abstract}
Cloud computing is emerging as widely accepted technology. It reduces need for high speed hardware, upgraded software and physical premises to carry out business or research activity. Virtualization in cloud supports sharing of resources to different users with different needs.
\end{abstract}

Cloud computing as an open forum, has created some security problems like confidentiality, integrity, and authentication. People need to be sure that their all communication on cloud is kept confidential. When they send their transactions request on cloud, they want to be certain that the integrity and security of the message is preserved.

It is prerequisite to ensure that user can access cloud data without worrying about security attacks. The most general tool for providing cloud security is cryptography, an old technique that has been revived and adopted by cloud computing.

The purpose of this research paper is to show how cloud platform is used successfully to store numerical data in the best protected manner. This paper discusses cloud computing present's security and confidentiality issues while applying numerical data in cloud. This paper also discusses current encryption schemes for cloud computing and recent developments in cryptosystems which can be implemented in cloud. We have also discussed about traditional and proposed methods to be applied for security of numerical data in cloud. Experiment and results of experiment are presented in the last section of this paper. At end, the paper concludes with advantages, applications and future research scope.

Keywords-cloud computing, encryption schemes, cryptosystem

\section{INTRODUCTION}

There are many applications which deal with numerical data and this data includes customer financials, account information, transactions and non public information. Much financial information generated or used by an industry is of sensitive and private. There is a need to protect sensitive information wherever it resides. Apart from security industry must fulfill the additional data security issues that result from normal operations.

\section{A. Security challenges of numerical data in cloud}

There are some important security challenges faced by cloud user when they deal with numerical data in cloud.

- Securely saving critical numerical data with maximum return and minimum risk.

- Allowing user to perform calculation on encrypted data without decrypting it. 
- Securing financial data not only during data rest but also during transmission of data.

- Single framework for data at rest protection: common need is to protect data at the file system and database level irrespective of the environment. The result - low total cost of ownership as well as simple efficient deployment and operations.

- To fulfill global compliance and regulatory standards.

- Cloud provider should meet for industry and governing standards while protecting information with this across the industry, cloud and big data environment.

\section{B. Confidentiality issue of numerical data in cloud}

Data sent to the cloud is stored at multiple data centre. This data centers are located at different places to serve user requirements anytime, anywhere. Following are the issues corresponding to cloud storage.

- Data sent in the cloud is stored in public cloud storage area.

- User's data should be controlled and managed by third party service providers.

- User has no control on data stored in cloud.

- Data may not be encrypted by cloud provider as it increases processing time of the file.

- Same key may be used to encrypt all customer data.

- It is not possible to search and index encrypted data.

- Encrypted data should be decrypted by third party cloud provider before performing calculation on it.

Encryption is adopted by most of the cloud providers for providing security and confidentiality of data in cloud provides only basic functionality like store and retrieve data in cloud but does not allow numerical operations on encrypted data. To perform operations on encrypted data should be decrypted first [ HYPERLINK \l "TEB13" 1 ].

As increased demand for security and more facility on cloud data several new encryption algorithms like order preserving and homomorphic encryption scheme are suggested over traditional encryption method in cloud. Till today people are looking for more improved and fully supportive technology.

An order preserving encryption scheme is an encryption algorithm that preserve numerical ordering of the plaintext after encrypting it. It supports range of query processing directly on encrypted data without decrypting them 2]. Homomorphic encryption allows us to perform mathematical operations on encrypted data and producing same answer as it is performed on unencrypted data.

At present AES is most approved standard for data security [ HYPERLINK $\backslash$ "Haa13" 3 ] but it does not provide calculation on encrypted numerical data. The solution to this problem is adoption of homomorphism encryption to save numerical data on cloud.

AES is extensively used in practice to secure application data in the cloud. While AES is a conventional encryption scheme to store data on cloud, it has many limited application functionalities such as search, logical operations and mathematical calculation. AES has also scalability problem in cloud environment 4].

\section{RELATED WORK}

Yunchuan Sun, Junsheng Zhang et. al. have focused on various issues of cloud computing like data confidentiality, data integrity, data availability and data privacy. Their research says that, homomrophic encryption ensures confidentiality of data and their implementation can solve problem of confidentiality of data and data operation in cloud [ HYPERLINK $\backslash 1$ "Yun14" 5 ]. 
Abha Sachdev, Mohit Bhansali proposed a simple data protection using Advance encryption standard for ensuring data confidentiality and security CITATION Abh13 $\backslash 1033$ [ HYPERLINK $\backslash 1$ "Abh13" 6 ] .

Maha TEBAA and Said EL HAJII have study the application of different Homomorphic Encryption cryptosystems like RSA, El Gamal, Goldwasser-Micali, Paillier, Boneh-Goh-Nissim and Gentry on a Cloud Computing platform. They compared different algorithms on bases of parameter like Homomorphic Encryption type, Privacy of data, Security applied and keys used CITATION TEB13 $\backslash 1033$ [ HYPERLINK $\backslash 1$ "TEB13" 1 ] .

Chungsik Song, Younghee Park reviewed a number of symmetric, public key and homomorphic cryptosystems to develop understanding of various encryption schemes for data on cloud storage. They suggested that AES is most secure encryption scheme for data on cloud storage. They also proved that Fully homomorphic encryption schemes are promising for cloud environment but it is practically difficult to implement because of their performance CITATION Chu15 $\backslash 1033$ [ HYPERLINK $\backslash 1$ "Chu15" 7 ] .

By Dr. Prerna Mahajan \& Abhishek Sachdeva analyzed that compare to RSA and DES, AES takes less encryption time. They also proved that decryption time is higher in RSA compare to AES and DES CITATION ByD13 $\backslash 1$ 1033 [ HYPERLINK \1 "ByD13" 8 ] .

HanumanthaRao.Galli and Dr.P.Padmanabham proposed a new security scheme to store data on cloud. They suggested s hybrid encryption and digital signature scheme for data security on cloud. In proposed system integrity of the data can be attained by creating message digest using MD5 algorithm. Blowfish algorithm is used to encrypt data for providing confidentiality. RSA algorithm is used for authentication CITATION Han13 $\backslash 1033$ HYPERLINK $\backslash 1$ "Han13" 9 ] .

\section{DIFFERENT ENCRYPTION METHODS IN CLOUD}

In this section we have discussed two encryption methods for cloud data storage AES encryption, Homomorphic encryption methods in cloud.

\section{AES Encryption}

AES is a symmetric key symmetric block cipher. AES is efficient for both hardware and software. AES supports block length of 128 bits and key lengths of 128,192 and 256 bits. AES is stronger and faster than 3DES. AES is an iterative cipher. AES is based on substitution and permutation network. AES performs all calculations on bytes than bits for example 128 bits of plain text is considered as block of 16 bytes. And these 16 bytes are arranged in matrix of four rows and four columns. Unlike DES number of rounds in AES depends on key length. There are 10 rounds for 128-bit keys, 12 rounds for 192-bit keys and 14 rounds for 256-bit keys. Each round uses 128 bit round key which is calculated from AES key CITATION Kak16 \1 1033 [ HYPERLINK \1"Kak16" 10 ] .

Nowadays AES is widely used. Till now no practical cryptanalytic attack against AES has been recorded. In addition AES has flexibility of key length CITATION Kak16 \1 1033 [ HYPERLINK \ "Kak16" 10 ] .

Major drawback with AES encryption is that it provides only secure storage but no calculation is allowed on encrypted data.

\section{Homomorphic Encryption}

Homomorphic encryption permits to perform complex numerical operation on encrypted data with no need to decrypt the encrypted numerical values. In mathematics, homomorphic express the alteration of one data set into 
another even after conserving relationships between elements mutually CITATION Sig11 11033 [ HYPERLINK l1 "Sig11" 11 ] .

There are two kinds of homomorphic encryption first is fully homomorphic encryption and second is somewhat homomorphic encryption. Fully homomorphic method supports unlimited number of addition and multiplication to be performed on encrypted data while somewhat homomorphic encryption support limited number of addition and multiplication on encrypted data. Somewhat homomorphic encryption is 30 years old idea but fully homomorphic encryption was invented in 2009. Researcher finds, it is difficult to use fully homomorphic encryption for today's application because high complexity of computations. Researcher hope that the drawback of fully homomorphic encryption should be addressed and practical solution could be implemented within a decade CITATION Res14 $\backslash$ 1033 [ HYPERLINK $\backslash$ "Res14" 12 ] .

Homomorphic encryption is anticipated to play a significant part in cloud computing, it permits business to store encrypted data in a cloud and take benefit of the cloud provider's analytic services CITATION Nas $15 \backslash 1033$ [ HYPERLINK $\backslash$ "Nas15" 13 ] .

A Homomorphic encryption has different Homomorphic schemes according to its Properties CITATION TEB13 $\backslash 1$ 1033 [ HYPERLINK \1 "TEB13" 1 ] :

TABLE I. HOMOMORPHIC ENCRYPTION SCHEMES

\begin{tabular}{|l|l|l|}
\hline $\begin{array}{l}\text { Different Homomorphic } \\
\text { Scheme }\end{array}$ & $\begin{array}{l}\text { Algorithm } \\
\text { Type }\end{array}$ & $\begin{array}{l}\text { Homomorphic } \\
\text { properties }\end{array}$ \\
\hline RSA & Asymmetric & Multiplicative \\
\hline Elgaml & Asymmetric & Multiplicative \\
\hline Goldwasser Micali & Asymmetric & XOR \\
\hline Benalh & Symmetric & Additive \\
\hline Paillier & Asymmetric & Additive \\
\hline Okamoto uchiyama & Asymmetric & Additive \\
\hline
\end{tabular}

\section{PROPOSED WORK}

In our proposed work we have discussed functioning of traditional method and homomorphic encryption method for data storage in cloud.

\section{Traditional Method}

When the data uploaded on cloud we use standard encryption methods to secure that data on cloud. Our basic concept is to encrypt the data at our premises before send it to the Cloud for storage. But at last one needs to decrypt data at every operation. In traditional method if client need to perform any calculations on encrypted data then he or she has to provide the private key to the cloud provider to decrypt data to convert them into plain text format to carry out calculations on it, once operation is performed data will be encrypted back and stored on cloud. This process of decryption and encryption is a challenge to the concept of confidentiality and privacy of data on Cloud. 
Encryption is a one solution to store the information securely on cloud but the main drawback is you need to have decryption key before searching or indexing your information. There are many encryption algorithm are available but encryption with homomorphic property is best suited in cloud environment.

\section{Homomorphic Encryption Method}

Homomorphic Encryption systems are used to carry out operations on encrypted data with no knowledge of private key (without decryption), the client is the merely holder of the secret key. When we decrypt the outcome of any operation, it is the same as if we had performed the calculation on the raw data.

Till homomorphic encryption look advantageous but usage of homomorphic encryption is limited, the reason is, it increases the size of encrypted data. Due to that reason data becomes so bulky to store it in a cloud. But in this research experiment it shows that homomorphic encryption is better in many ways compare to traditional encryption method for numerical data.

\section{EXPERIMENTAL SETUP AND TESTING}

The implementation outcome are taken on machine having Intel ${ }^{\circ}$ Core ${ }^{\mathrm{TM}}$ i5-3210M CPU @ $(2.50 \mathrm{GHz})$ processor with Installed memory(RAM) 4.00GB and 64-bit Operating System. The java platform JDK 7u80 with NetBeans 8.0.2 is used for implementation. JCA (Java Cryptography Architecture) and JCE (Java Cryptography Extension) are used for cipher algorithm implementation. A timer is started before encrypting or decrypting a value. After encryption or decryption is performed, the timer is stopped. Due to this pure encryption time is obtained for assessing the performance of a particular cipher algorithm.

TABLE II. COMPARISON BETWEEN AES AND PAILLIER HOMOMORPHIC ENCRYPTION

\begin{tabular}{|c|c|}
\hline AES Encryption & Paillier Homomorphic Encryption \\
\hline $\begin{array}{l}\text { Plain Text before Encryption: } 25 \text { (First } \\
\text { Value or F.V.) } \\
\text { Plain Text before Encryption: } 60 \text { (Second } \\
\text { Value or S.V.) }\end{array}$ & $\begin{array}{l}\text { Plain Text before Encryption: } 25 \text { (First Value or F.V.) } \\
\text { Plain Text before Encryption: } 60 \text { (Second Value or S.V.) }\end{array}$ \\
\hline $\begin{array}{l}\text { AES Encryption time in millisecond (F.V.) } \\
: 40 \\
\text { AES Encryption time in millisecond (S.V.) } \\
: 0\end{array}$ & $\begin{array}{l}\text { Homomorphic Encryption time in millisecond (F.V.):11 } \\
\text { Homomorphic Encryption time in millisecond (S.V.):10 }\end{array}$ \\
\hline $\begin{array}{l}\text { AES Encrypted Text After Encryption } \\
\text { (F.V.): oA8y8CVvbMm69yobmqsGRQ== } \\
\text { AES Encrypted Text After } \\
\text { Encryption(S.V.): } \\
\text { 6+YfHJ394C0Po0J39Te3iw== }\end{array}$ & $\begin{array}{l}\text { Homomorphic Encrypted Text (F.V.): } \\
68008499469075871995379066135399269734600831493695394451 \\
87715636027657422850262132233619910187516128067560614831 \\
99411952829298139436681322478839781124708473881993851245 \\
88390421367673602197123029413967421125145892893875489280 \\
11114318529052328184141031593540790307599640185539147383 \\
456700740580951898713744676 \\
\text { Homomorphic Encrypted Text (S.V.): } \\
89553158224512213683853571133379547652425130881219650666 \\
53934308885634670342794046269581169358597762147833795401 \\
42761591797722928151966813891692073463722036184597474838\end{array}$ \\
\hline
\end{tabular}




\begin{tabular}{|l|l|}
\hline & 87156538569913308610910572785668427466207551035271006087 \\
& 42296436735600865284190761470493962529815277657807348177 \\
& 164667752064759550426534719 \\
\hline
\end{tabular}

TABLE III. COMPARISON BETWEEN PLAIN NUMBERS AND PAILLIER HOMOMORPHIC ENCRYPTED NUMBERS MATHEMATICAL OPERATIONS

\begin{tabular}{|c|c|}
\hline Plain Numbers & Homomorphic Encrypted Numbers \\
\hline Numbers: 25 and 60 & $\begin{array}{l}\text { Homomorphic Encrypted Text for } 25: \\
68008499469075871995379066135399269734600831493695394451877156 \\
36027657422850262132233619910187516128067560614831994119528292 \\
98139436681322478839781124708473881993851245883904213676736021 \\
97123029413967421125145892893875489280111143185290523281841410 \\
31593540790307599640185539147383456700740580951898713744676 \\
\text { Homomorphic Encrypted Text for } 60: \\
89553158224512213683853571133379547652425130881219650666539343 \\
08885634670342794046269581169358597762147833795401427615917977 \\
22928151966813891692073463722036184597474838871565385699133086 \\
10910572785668427466207551035271006087422964367356008652841907 \\
61470493962529815277657807348177164667752064759550426534719\end{array}$ \\
\hline $\begin{array}{l}\text { Mathematical Operation: Addition } \\
\text { Answer: } 85\end{array}$ & $\begin{array}{l}\text { Mathematical Operation: Addition } \\
\text { Answer: } 85\end{array}$ \\
\hline $\begin{array}{l}\text { Mathematical Operation time in } \\
\text { millisecond: } 0\end{array}$ & Mathematical Operation time in millisecond: 0 \\
\hline $\begin{array}{l}\text { Mathematical Operation: } \\
\text { Multiplication } \\
\text { Answer: } 1500\end{array}$ & $\begin{array}{l}\text { Mathematical Operation: Multiplication } \\
\text { Answer: } 1500\end{array}$ \\
\hline $\begin{array}{l}\text { Mathematical Operation time in } \\
\text { millisecond: } 0\end{array}$ & Mathematical Operation time in millisecond: 0 \\
\hline
\end{tabular}

The result for the comparison demonstrate that Homomorphic is not an expensive encryption it has obvious advantages over AES encryption like less encryption time, and equal time for mathematical operations as normal plain text calculations. This experiment has been carried out many times and every time it reflects that homomorphic encryption is taking less time compare to AES encryption. But only drawback is Homomorphic ciphertexts are significantly larger than the plaintexts.

TABLE IV. COMPARISON BETWEEN AES-128 ENCRYPTION AND HOMOMORPHIC ENCRYPTION

\begin{tabular}{|c|c|c|}
\hline PARTICULAR & AES ENCRYPTION & $\begin{array}{c}\text { HOMOMORPHIC } \\
\text { ENCRYPTION }\end{array}$ \\
\hline ENCRYPTION TIME & HIGH & LOW \\
\hline CIPHER TEXT SIZE & SHORT & LONG \\
\hline
\end{tabular}


TABLE V. COMPUTATION TIME FOR MATHEMATICAL OPERATIONS

\begin{tabular}{|c|c|c|}
\hline PARTICULAR & PLAIN NUMBER & $\begin{array}{c}\text { HOMOMORPHIC } \\
\text { ENCRYPTION NUMBER }\end{array}$ \\
\hline $\begin{array}{c}\text { MATHAMATICAL OPERATION } \\
\text { TIME }\end{array}$ & SAME & SAME \\
\hline
\end{tabular}

Homomorphic encryption can be used for many numerical based encryption systems few of them are online voting, online feedback, online survey, marketing analysis, and salary payment. We can still explore many homomorphic encryption supported applications.

\section{CONCLUSIONS}

The selection of an encryption scheme depends on the competence and security requirement of the application used. It has been observed that there is no single encryption system basically suited for all types of data and different purposes to store data on cloud. However, Homomorphic encryption schemes show potential in terms of security but they lack in term of efficiency in encrypting large number of data as compared to AES schemes. Day by day challenge increases to construct more secure and efficient cloud data storage. Homomorphic encryption is a hopeful idea for providing security for many calculations based applications but there is still lot of scope of improvements and analysis of these schemes required to be done for practical implementation. In future our focus would be on designing and developing cloud based secure and efficient real time cloud applications using homomorphic encryption.

\section{REFERENCES}

[1] Maha TEBAA and Said EL HAJII, "Secure Cloud Computing through Homomorphic Encryption," International Journal of Advancements in Computing Technology(IJACT), vol. 5, December 2013. [Online]. HYPERLINK "http://arxiv.org/ftp/arxiv/papers/1409/1409.0829.pdf" http://arxiv.org/ftp/arxiv/papers/1409/1409.0829.pdf

[2] Alexandra Boldyreva, Nathan Chenette, Younho Lee, and Adam O’Neill, "Order-Preserving Symmetric Encryption," in Advances in Cryptology - Eurocrypt 2009 Proceedings, vol. Computer Science Vol. 5479, Germany, April 2009. [Online]. HYPERLINK "http://www.cc.gatech.edu/ aboldyre/papers/bclo.pdf" http://www.cc.gatech.edu/ aboldyre/papers/bclo.pdf

[3] Keijo Haataja, Konstantin Hyppönen, Sanna Pasanen, and Pekka Toivanen, Bluetooth Security Attacks.: Springer-Verlag Berlin Heidelberg, 2013. [Online]. HYPERLINK "http://www.springer.com/in/book/9783642406454" \l "aboutAuthors" http://www.springer.com/in/book/9783642406454\#aboutAuthors

[4] Chungsik Song, Younghee Park, Jerry Gao, Sri Kinnera Nanduri, and William Zegers, "Favored Encryption Techniques and Evaluation for Big Data in a Cloud," in The FIRST IEEE INTERNATIONAL CONFERENCE on Big Data Computing Service, and Applications, San Francisco Bay, California, USA, MARCH 2015

[5] Junsheng Zhang, Yongping Xiong, and Guangyu Zhu Yunchuan Sun, "Data Security and Privacy in Cloud Computing," International Journal of Distributed Sensor Networks, vol. 2014 (2014), no. 16 July, July 2014.

[6] Mohit Bhansali Abha Sachdev, "Enhancing Cloud Computing Security using AES Algorithm," International Journal of Computer Applications (0975 - 8887), vol. 67- No.9, April 2013

[7] Younghee Park, Jerry Gao, Sri Kinnera Nanduri, William Zegers Chungsik Song, "Favored Encryption Techniques for Cloud Storage," in IEEE, Redwood City, CA, 2015, pp. $267-274$

[8] Abhishek Sachdeva By Dr. Prerna Mahajan, "A Study of Encryption Algorithms AES, DES and RSA for," Global Journal of Computer Science and Technology, vol. 13, no. 15, 2013

[9] Dr.P.Padmanabham HanumanthaRao.Galli, "Data Security in Cloud using Hybrid Encryption and Decryption," International Journal of Advanced Research in Computer Science and Software Engineering, vol. 3, no. 10, pp. 494-497, October 2013

[10] Avi Kak. (2016, February ) https://engineering.purdue.edu. [Online]. "https://engineering.purdue.edu/kak/compsec/NewLectures/Lecture8.pdf" https://engineering.purdue.edu/kak/compsec/NewLectures/Lecture8.pdf

[11] Sigrun Goluch, "The development of," Discrete Mathematics and Geometry Vienna University of Technology, Wien, Austria, T H E S I S 2011.

[12] Research Directorate Staff, "Securing the cloud with homomorphic encryption," The Next Wave (TNW), vol. 2, no. 3, 2014. [Online]. HYPERLINK "https://classes.soe.ucsc.edu/cmps122/Fall15/content/TNW203_article5.pdf" https://classes.soe.ucsc.edu/cmps122/Fall15/content/TNW203_article5.pdf

[13] Ahmed Kayed Nasrin Dalil, "Preserving Data in Cloud Computing ," International Journal of Computer Science Issues, vol. 12, no. 2, pp. 296-300, March 2015. 